ARTICLE

Received 6 Aug 2016 | Accepted 3 Jan 2017 | Published 16 Feb 2017

\title{
Superconductivity in a chiral nanotube
}

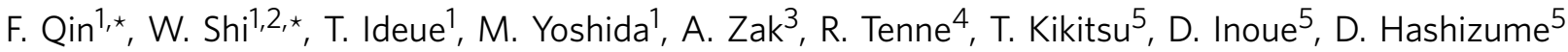 \\ \& Y. Iwasa ${ }^{1,5}$
}

Chirality of materials are known to affect optical, magnetic and electric properties, causing a variety of nontrivial phenomena such as circular dichiroism for chiral molecules, magnetic Skyrmions in chiral magnets and nonreciprocal carrier transport in chiral conductors. On the other hand, effect of chirality on superconducting transport has not been known. Here we report the nonreciprocity of superconductivity-unambiguous evidence of superconductivity reflecting chiral structure in which the forward and backward supercurrent flows are not equivalent because of inversion symmetry breaking. Such superconductivity is realized via ionic gating in individual chiral nanotubes of tungsten disulfide. The nonreciprocal signal is significantly enhanced in the superconducting state, being associated with unprecedented quantum Little-Parks oscillations originating from the interference of supercurrent along the circumference of the nanotube. The present results indicate that the nonreciprocity is a viable approach toward the superconductors with chiral or noncentrosymmetric structures.

\footnotetext{
${ }^{1}$ Quantum-Phase Electronics Center (QPEC) and Department of Applied Physics, The University of Tokyo, Tokyo 113-8656, Japan. ${ }^{2}$ Materials Sciences Division, Lawrence Berkeley National Laboratory, Berkeley, California 94720, USA. ${ }^{3}$ Faculty of Sciences, Holon Institute of Technology, 52 Golomb Street, PO Box 305, Holon 58102, Israel. ${ }^{4}$ Department of Materials and Interfaces, Weizmann Institute of Science, Rehovot 76100, Israel. ${ }^{5}$ RIKEN Center for Emergent Matter Science (CEMS), Wako, Saitama 351-0198, Japan. * These authors contributed equally to this work. Correspondence and requests for materials should be addressed to T.I. (email: ideue@ap.t.u-tokyo.ac.jp).
} 
C hirality of crystal or magnetic structures in solids was recently recognized as a powerful source of unique optical and electronic properties and novel functionalities. For instance, it is well known that polarization of light or spin of electron are sensitive to the chirality of lattice or magnetic structure $^{1-4}$ and electric transport reflecting the chiral structure are also reported ${ }^{5-7}$. Among them, effects of chiral structures on superconductivity have not been investigated so far because of the lack of suitable materials. One of the interesting superconducting materials with chirality is carbon nanotube $(\mathrm{NT})^{8,9}$. Chiral structures and their relations to the electronic properties in carbon NTs have been well studied by Raman scattering ${ }^{10,11}$, scanning tunnel microscope ${ }^{12}$ and even magneto-chiral transport $^{6}$. However, since superconductivity in carbon NTs ${ }^{13-16}$ has been investigated only in the assembled form of single, double or multi-walled NTs, relations between superconducting transport and the chirality in individual tubes have remained elusive.

Tungsten disulfide $\left(\mathrm{WS}_{2}\right)$ is a member of transition metal dichalcogenides (TMDs), which are now attracting significant attention as two dimensional (2D) materials beyond graphene with the potential application for electronics, photonics, spintronics, mechanics, as well as valleytronics ${ }^{17,18}$. Recent systematic studies clarified many TMDs including $\mathrm{WS}_{2}$, which are semiconductors without carrier doping, exhibit superconductivity under the ionic gating ${ }^{19,20}$. Importantly, TMD can form tubular structures with noncentrosymmetric chiral structures ${ }^{21-26}$. The semiconducting property of the $\mathrm{WS}_{2} \mathrm{NT}$ was indeed demonstrated using the field effect transistor devices ${ }^{24}$. Superconductivity in such a noncentrosymmetric chiral cylinder, once it is realized, is a potential candidate for searching the exotic quantum phenomena and nontrivial Cooper pairing ${ }^{27,28}$. One of the manifestations of the chiral structure in the electronic transport is the unidirectional resistance. As shown in Fig. 1a,b, the two directions of current injection are not identical due to the chiral nature of the conducting substance when the magnetic field is applied parallel to the tube. Such nonreciprocity is highly anticipated to yield nontrivial quantum transport particularly in the superconducting states.

In this study, the transport properties of individual $\mathrm{WS}_{2} \mathrm{NT}$ have been investigated by using the ionic liquid gating technique and resistance measurement on both first and second harmonic signals in alternative current (AC) mode. We have observed ambipolar transfer curve in electrostatic doping region and the emergence of superconductivity by electrochemical doping. The superconducting properties of individual $\mathrm{WS}_{2} \mathrm{NT}$ have been further investigated, in which the observed anisotropy of the superconductivity and Little-Parks (LP) oscillation $^{29}$ are consistent with tubular structure of $\mathrm{WS}_{2}$ NT. More importantly, we have experimentally discovered nonreciprocal superconducting transport via the second harmonic signal, being suggestive of chirality effect on superconductivity. Such nonreciprocal signal is largely enhanced in the superconducting state and affected by the magnetic flux quantum, showing periodic oscillations. The present study paves a route for studying the interplay between superconductivity and chirality or noncentrosymmetry.

\section{Results}

Sample characterization. $\mathrm{WS}_{2}$ NTs were synthesized following the literature ${ }^{21-23}$. Figure 1c shows a transmission electron microscope (TEM) image of a single $\mathrm{WS}_{2}$ NT (see Supplementary Fig. 1). The tube has a multi-walled structure, with the outer/ inner diameters estimated as $132 / 107 \mathrm{~nm}$, respectively, indicating that the layer number is $\sim 20$. According to the literature ${ }^{21-23}$, the tube part has a $2 \mathrm{H}$-polymorph-layered structure of $\mathrm{WS}_{2}$, where each tungsten atom is surrounded by six sulfur atoms in a trigonal biprism coordination (space group $P 63 / \mathrm{mmc}$ ). The outer diameter distribution of tubes in the batch used for this measurement takes a broad maximum around $100 \mathrm{~nm}$ (See Supplementary Fig. 4). An electron diffraction pattern of a single $\mathrm{WS}_{2} \mathrm{NT}$ is displayed in Fig. 1d. The red arrow and yellow hexagon represent the direction of tube axis and diffraction a

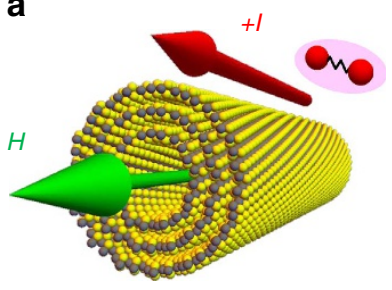

b

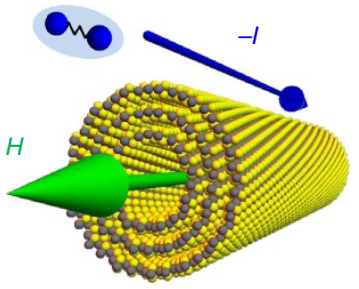

C

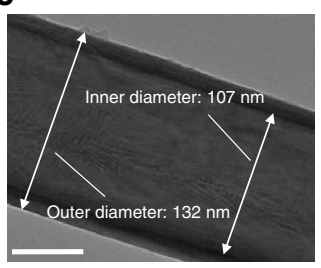

d

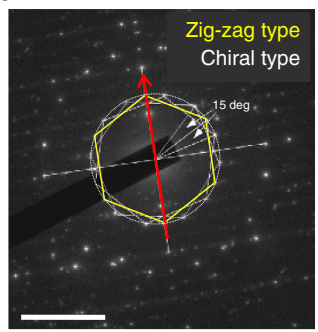

e

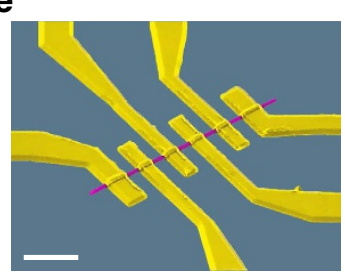

f

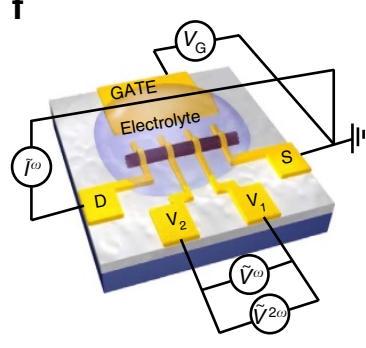

Figure 1 | WS $\mathbf{2}$ chiral NT and asymmetric superconducting transport in chiral NTs. (a,b) Illustrations of the unidirectional electric transport in superconducting chiral NT. Because of the broken inversion symmetry, asymmetric magnetoresistance is expected under magnetic field parallel to the tube axis, which can be probed via second harmonic signals of AC resistance. (c) TEM picture of a single WS 2 NT. Contrast image shows the cylindrical structure with a diameter of about $100 \mathrm{~nm}$. Scale bar, $50 \mathrm{~nm}$. (d) Electron diffraction pattern of a single WS $\mathrm{W}_{2}$ NT. Scale bar, $5 \mathrm{~nm}{ }^{-1}$. The red arrow represents the direction of the tube axis. Tilted hexagonal diffraction pattern (white lines) confirms the existence of the chiral structure in addition to the zigzag type NT (yellow line) (see Supplementary Figs 5-9). (e) Coloured scanning electron microscope image (SEM) of a WS 2 NT device. Scale bar, $2 \mu$ m. (f) Sketch of the electric double-layer transistor device. The electrolyte $\mathrm{KClO}_{4}$ /polyethylene glycol is used as the gate medium. Both the first and second harmonic signals of the AC resistance have been measured by lock-in amplifiers (see 'Methods' section). 
pattern from the zigzag type NT, respectively. The different walls of the tube can have different chirality. In addition to the contribution of the zigzag type NT, we can see the pair of tilted hexagonal pattern which confirms the co-existence of chiral structures in this NT. The TEM analysis indicates that the tubes used for the transport measurement are multi-walled $\mathrm{WS}_{2} \mathrm{NTs}$ with chirality, having the outer diameter of nearly $100 \mathrm{~nm}$ (See Supplementary Fig. 4).

Ionic liquid gating on $\mathrm{WS}_{2} \mathrm{NT}$ and superconductivity. We fabricated an individual tube device as shown in Fig. 1e,f, and measured gate responses of the transport characteristics. Based on our previous research on the systematic study of superconductivity in $\mathrm{TMDs}^{19}$, we used $\mathrm{KClO}_{4} /$ polyethylene glycol electrolyte as the gate medium to facilitate electrochemical intercalation of potassium ions into the layered structure of $\mathrm{WS}_{2}$.

Figure 2a displays the source-drain current $\left(I_{\mathrm{DS}}\right)$ of the individual $\mathrm{WS}_{2} \mathrm{NT}$ device against the gate voltage $\left(V_{\mathrm{G}}\right)$ between -2 and $3 \mathrm{~V}$. The device nicely operates in an ambipolar mode, in a similar manner to the $2 \mathrm{D}$ devices, showing marked contrast with the unipolar response of $\mathrm{WS}_{2}$ NTs in the solid gated field effect transistor ${ }^{24}$. This indicates the strong gate coupling of the presently used ionic medium. The transistor operation is most likely in the electrostatic mode in this regime, considering the ambipolar behaviour is reversible and repeatable. When $V_{\mathrm{G}}$ was increased to $8 \mathrm{~V}$ at a constant rate of $50 \mathrm{mV} \mathrm{s}^{-1}$, we found a saturation of $I_{\mathrm{DS}}$, similarly to the case of $2 \mathrm{D} \mathrm{WS}{ }_{2}$ (ref. 19). When $V_{\mathrm{G}}$ was kept at $8 \mathrm{~V}$ for a couple of minutes, we encountered another dramatic increase of $I_{\mathrm{DS}}$ by more than two orders of magnitude as shown in Fig. $2 \mathrm{~b}$. This $I_{\mathrm{DS}}$ increase is presumably attributed to intercalation of $\mathrm{K}^{+}$ions into $\mathrm{WS}_{2}$ NTs.

When we cooled down the device to $2 \mathrm{~K}$ keeping $V_{\mathrm{G}}$ at $8 \mathrm{~V}$, superconductivity appeared at $T_{\mathrm{c}}=5.8 \mathrm{~K}$, defined as the temperature corresponding to the half of normal state resistance (Fig. 2c). In contrast to the K-intercalated $2 \mathrm{D} \mathrm{WS}_{2}$ multilayer with $T_{\mathrm{c}}$ of $8.6 \mathrm{~K}$ (ref. 19), the superconducting transition here is shifted to lower temperature and considerably broadened, potentially due to the reduced dimensions or lack of commensurability between the different walls.
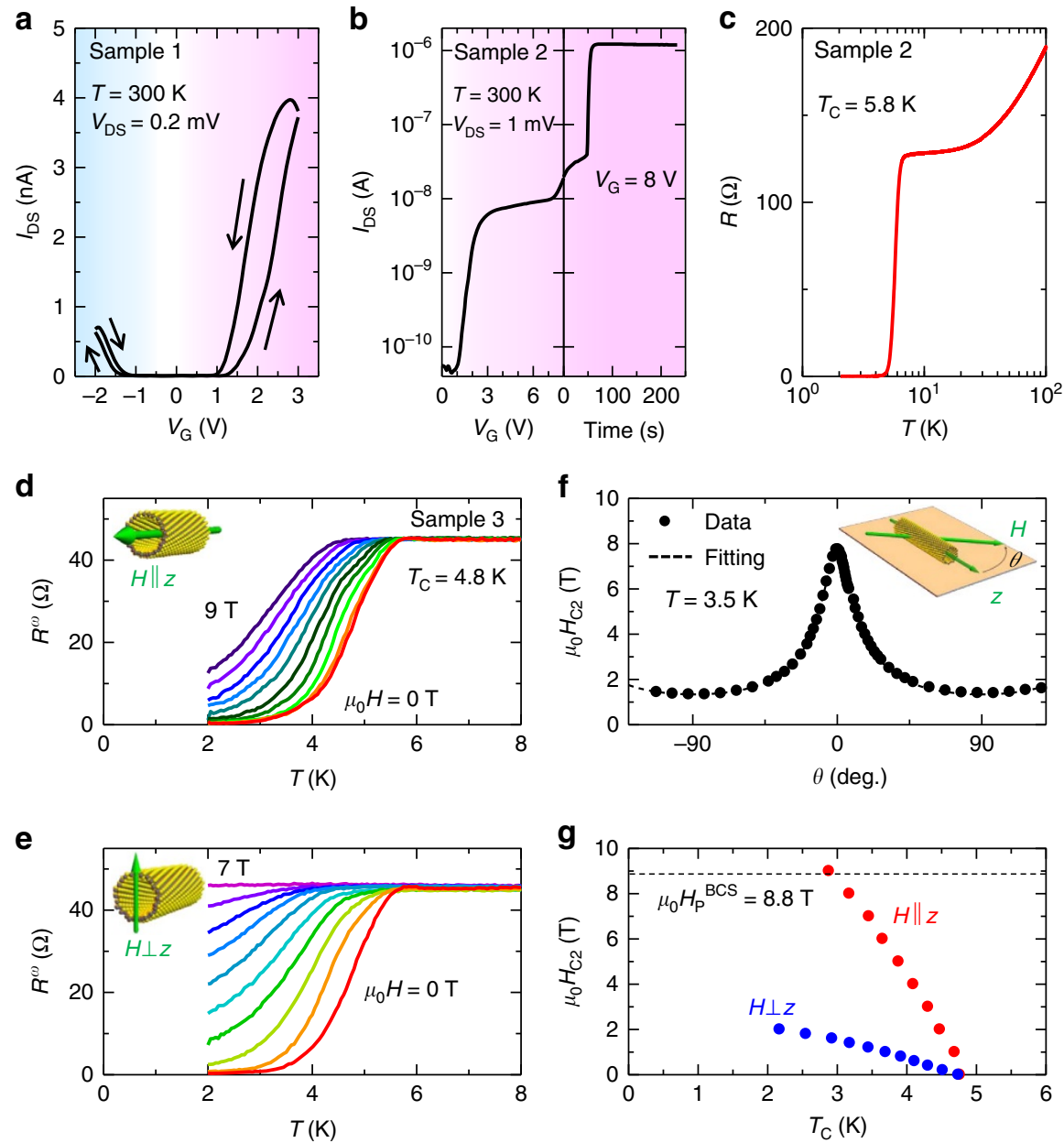

Figure 2 | lonic gating effect on $\mathbf{W S}_{\mathbf{2}}$ NT and anisotropic superconducting behaviour. (a) Ambipolar transfer curve $\left(I_{D S}\right.$ versus $\left.V_{G}\right)$ measured from sample 1. (b) $I_{D S}$ as a function of $V_{G}$ and waiting time measured from sample 2 . First and second increase of $I_{D S}$ observed at the electron-doped side can be attributed to the electrostatic and electrochemical doping, respectively ${ }^{19}$. (c) Superconducting transition after ionic gating with $V_{G}=8 \mathrm{~V}$. $T_{\mathrm{c}}$ is $5.8 \mathrm{~K}$, defined as the temperature corresponding to the half of the normal state resistance. (d,e) Temperature dependence of the resistance under magnetic field $H$ parallel (d) and perpendicular (e) to the tube axis $z$ measured from sample 3. (f) Angle dependence of the critical magnetic field $H_{\mathrm{c} 2}$ measured at $3.5 \mathrm{~K}$. Circles and dashed line represent the critical magnetic field obtained from the experiment and theoretical fitting by the anisotropic Ginzburg-Landau model. We define $\theta=0$ degree when the magnetic field is parallel to the tube axis. (g) Temperature dependence of the perpendicular and parallel critical magnetic field $H_{c 2}$. Dashed line indicates the Pauli paramagnetic limit. 

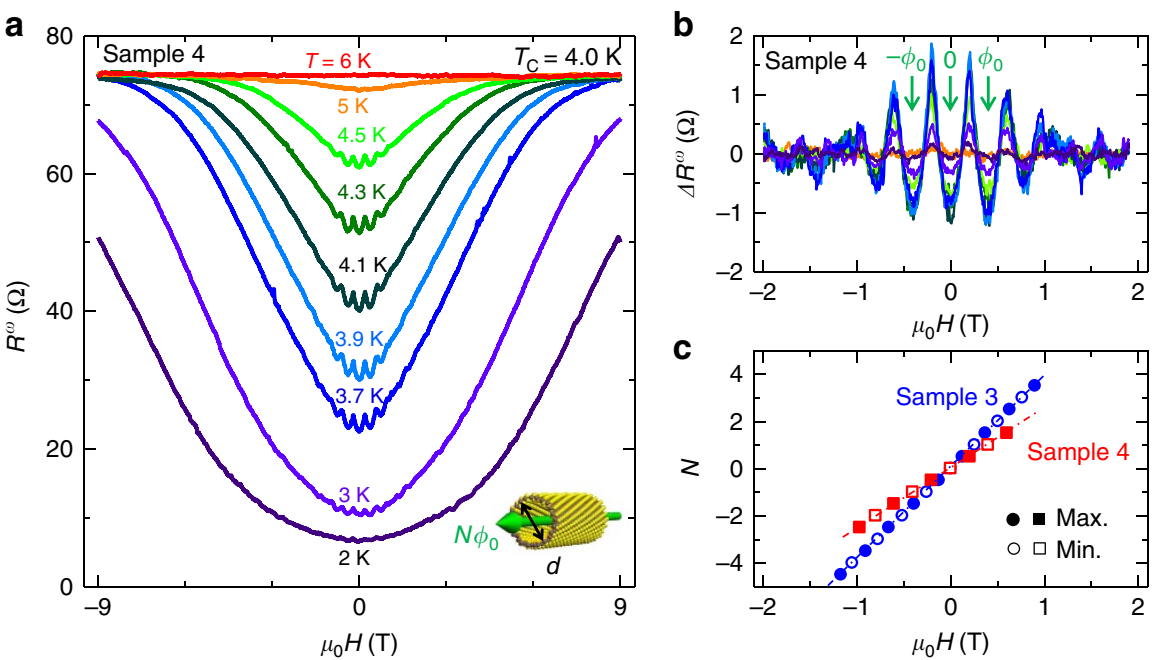

Figure 3 | Little-Parks oscillations in the superconducting phase of $\mathbf{W S}_{\mathbf{2}}$ NT. (a) Magnetoresistance measured from sample $4 . R^{\omega}$ denotes the first harmonic signal of the $A C$ resistance (normal $A C$ resistance measured by lock-in amplifier). LP oscillations appear when the magnetic field is applied along the tube axis (see inset cartoon), which indicates the interference of the superconducting current around the circumference. (b) Oscillating components calculated by subtracting the polynomial backgrounds from $\mathbf{a}$. The oscillation shows a period of $\phi_{\mathrm{O}}$ and enhanced magnitude around $T_{\mathrm{c}}$. (c) Index plot for the maximum and minimum peak positions of the LP oscillations. Open and closed circles (squares) correspond to the minimum and maximum positions for sample 3 (sample 4), respectively. We defined $N=0$ as the minimum peak position at zero magnetic field. From the slope of this plot, we can estimate the effective diameter $d$ of the superconducting region, which is consistent with the values obtained from TEM observation.

We then investigated the anisotropy of the observed superconductivity for sample $3\left(V_{\mathrm{G}}=6 \mathrm{~V}\right)$. Figure $2 \mathrm{~d}$,e displays the temperature variation of the resistance under magnetic field $H$ for $H \perp z$ and $H \| z$, respectively. Here $z$ represents the tube axis direction. In the case of the $H \| z$, the superconductivity is robust against the magnetic field and remains undefeated even under $\mu_{0} H=9 \mathrm{~T}$ at $T=2 \mathrm{~K}$, while the superconducting phase rapidly disappears for the $H \perp z$ configuration with increase of magnetic field. The anisotropic superconductivity was also confirmed by the angular dependence (Fig. 2f) and temperature dependence (Fig. 2g) of the upper critical magnetic field. In Fig. 2f, the estimated upper critical field at $T=3.5 \mathrm{~K}$ are well fitted by the anisotropic Ginzburg-Landau model $\mu_{0} H_{\mathrm{C} 2}=\frac{1}{\sqrt{(a \cos \theta)^{2}+(b \sin \theta)^{2}}}$ with fitting parameters $a=0.13 \mathrm{~T}^{-1}$ and $b=0.75 \mathrm{~T}^{-1}$. The temperature dependence of the critical magnetic field (Fig. 2g) cannot be explained either by the simple $2 \mathrm{D}$ or $3 \mathrm{D}$ models, implying that the system is of intermediate dimension. Here we should note that the upper critical field at $T=0 \mathrm{~K}$ for the $H \| z$ configuration seemingly exceeds the Pauli paramagnetic limit $\mu_{0} H_{\mathrm{P}}^{\mathrm{BCS}}=\frac{\sqrt{2}}{g \mu_{\mathrm{B}}} \Delta \sim 1.84 T_{\mathrm{c}}=8.8 \mathrm{~T}$ ( $\Delta$ is the superconducting gap at $T=0 \mathrm{~K}$ ), being suggestive of a strong spin-orbit interaction and nontrivial Cooper pairing in the present system (See Supplementary Note 2).

Little-Parks oscillations. Figure 3 a shows the AC magnetoresistance of sample $4\left(V_{\mathrm{G}}=12 \mathrm{~V}\right)$ at various temperatures around $T_{\mathrm{c}}$ in $H \| z$ configuration. In addition to the robustness of the superconductivity discussed above, the magnetoresistance observed via the first harmonic signals in AC resistance $\left(R^{\omega}\right)$ shows periodically oscillating behaviour in the low-magnetic field region. These oscillations during the superconducting transition known as LP effect ${ }^{29}$ originate from the interference of the superconducting current along the NT circumference and the resultant oscillations of $T_{\mathrm{c}}$ (refs 29,30). During the application of a parallel magnetic field, the total flux piercing the NT should have a quantized value of $N \phi_{0}=N h / 2 e$ and induce the oscillation of the free energy, manifested by the resistance oscillation with the period of $\phi_{0}=h / 2 e$ as observed in Fig. 3a ( $h$ and $e$ represent the Plank constant and charge of the electron, respectively, while $N$ is an integer representing the number of flux quantum). Similar LP oscillations have been also observed in sample 3 (See Supplementary Fig. 11). We plotted the oscillating components at different temperatures in Fig. $3 \mathrm{~b}$ after subtracting the polynomial background from Fig. 3a. The magnitude of the oscillating components reaches a maximum around $T_{c}$ (see also Fig. $4 \mathrm{f}$ ). From the periods of LP oscillations $\Delta\left(\mu_{0} H\right)$, we can estimate the effective diameter $d$ of the superconducting NT to be 100 and $80 \mathrm{~nm}$ for sample 3 and 4 , respectively, according to the relation $\frac{\Delta\left(\mu_{0} H\right) \cdot \pi d^{2}}{4}=\frac{h}{2 e}$ (Fig. 3c). This is consistent with the diameter distribution histogram of the same batch shown in Supplementary Fig. 4. These results provide firm evidence that superconductivity occurs in the tubular region in the present $\mathrm{WS}_{2}$ sample.

Nonreciprocal superconducting transport in chiral $\mathrm{WS}_{2} \mathrm{NT}$. To clarify the characteristic properties due to the chiral structure, we have measured the second harmonic signals in the AC resistance $\left(R^{2 \omega}\right)$. In noncentrosymmetric systems, the cross term of magnetic field $H$ and electric current $I$ in the resistance is allowed on the basis of the symmetry argument, which indicates the difference between the forward and backward transports under magnetic field ${ }^{5-7}$. This term generates the nonlinear voltage response, which can be measured as the second harmonic components in the AC resistance (See Supplementary Note 3).

Especially in chiral systems, this nonreciprocal electric transport called magneto-chiral anisotropy has been reported in several materials with different chiral degrees of freedom ${ }^{5-7}$. Phenomenologically, magneto-chiral anisotropy can be expressed as

$$
R^{2 \omega}=\frac{1}{2} \gamma R_{0} \mu_{0} H I
$$

where both the magnetic field $H$ and electric current $I$ are parallel to the chiral axis and $\gamma$ is the ratio of $R^{2 \omega}$ to the normal resistance $R_{0}$. So far, there has been no report of such a phenomenon in 
a

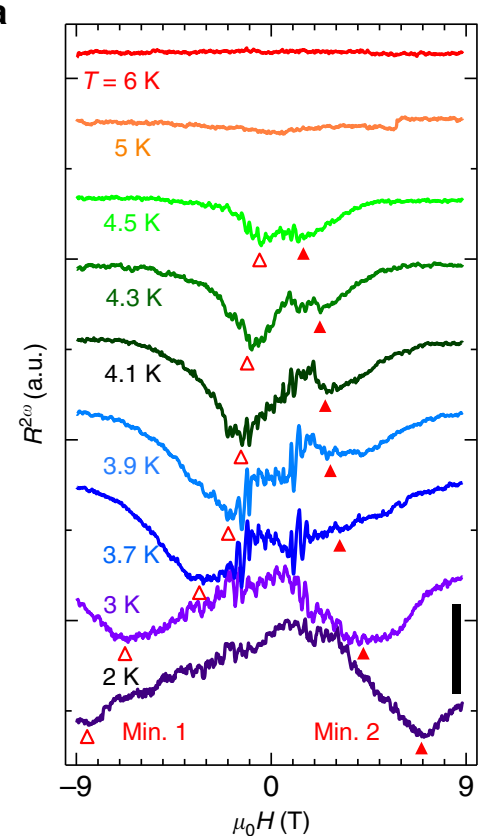

b

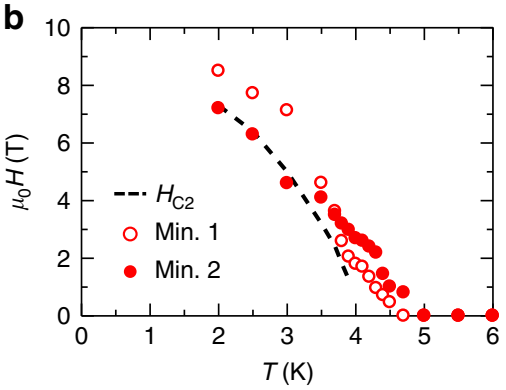

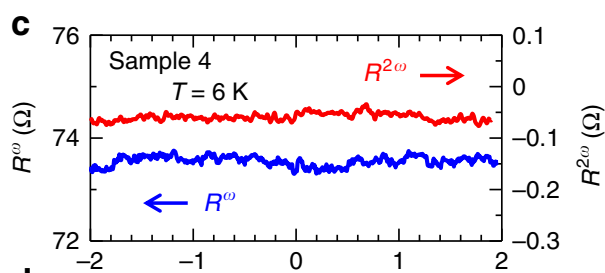

d 50

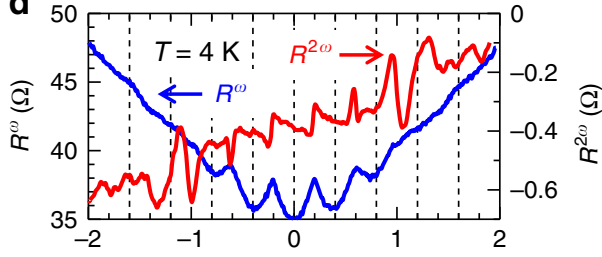

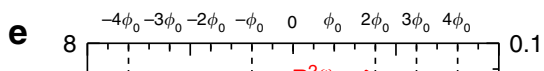
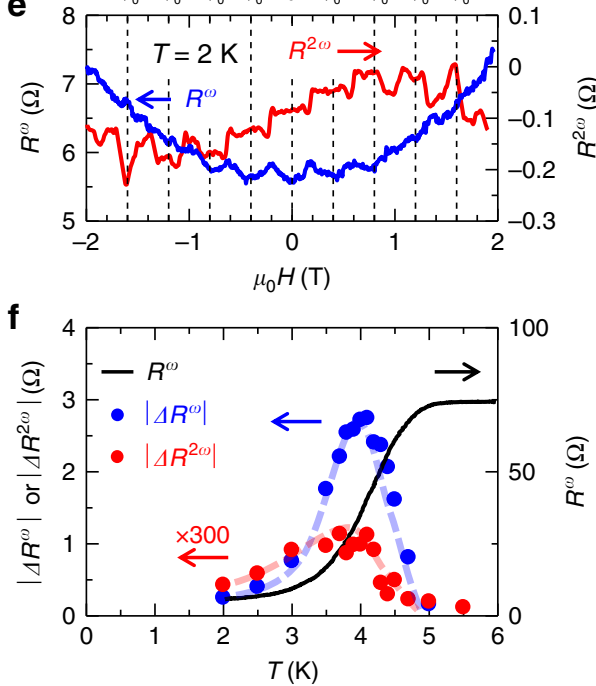

Figure 4 | Nonreciprocal superconducting transport probed by second harmonic signals in AC magnetoresistance. (a) Magnetic field dependence of the second harmonic components in the AC resistance $\left(R^{2 \omega}\right)$ measured from sample 4 . Scale bar, $0.5 \Omega$. Finite $R^{2 \omega}$ signals reflecting the symmetry breaking in the chiral structure are observed during the superconducting transition. (b) Temperature dependence of upper critical magnetic field $H_{\mathrm{c} 2}$ and characteristic minimum positions in $R^{2 \omega}$. Each set of minimum positions gradually changes with temperature, showing similar behaviour as the critical magnetic field $H_{\mathrm{c} 2}$. (c-e) Comparative plots of $R^{\omega}$ and $R^{2 \omega}$ in low-magnetic field region at $T=6,4$ and $2 \mathrm{~K}$. Periodic oscillations were observed for both signals in the superconducting state ( 2 and $4 \mathrm{~K}$ ), which disappears rapidly in the normal state $(6 \mathrm{~K}) . R^{2 \omega}$ shows the stepwise behaviour at low temperature $(2 \mathrm{~K})$. (f) Temperature variation of the resistance and magnitude of the quantum oscillations in $R^{\omega}$ and $R^{2 \omega}$. For $R^{2 \omega}$ oscillations, we estimate the magnitude of the jumps which are observed in low-field region. Both oscillating signals are enhanced around $T_{\mathrm{c}}$.

superconducting phase and it is of great interest whether excitation or quasiparticle in superconducting state without inversion symmetry generates such an unidirectional magnetoresistance.

Figure 4a shows the magnetic field dependence of second harmonic components $R^{2 \omega}$ in the AC resistance measured in sample 4. Finite antisymmetric $R^{2 \omega}$ signals were observed in the superconducting region, which unambiguously indicate the unidirectional electrical transport due to the chiral symmetry (Fig. 1a,b). In sharp contrast, $R^{2 \omega}$ signals are negligibly small in the normal state (see Fig. 4c), indicating that magneto-chiral anisotropy signals are significantly enhanced in the superconducting phase due to the coherent nature of superconductivity.

The observed $R^{2 \omega}$ signal has the two characteristic structures: that is, the broader antisymmetric components and the periodically oscillating terms in the low-magnetic field region. In the broader antisymmetric components, there are characteristic minima indicated by small triangles in Fig. 4a, which are dependent on temperature and enhanced at low temperature. The temperature dependence of the characteristic minima show similar behaviour as the critical magnetic field (Fig. 4b), strongly suggesting that the observed signals are related to the superconducting transition. (We show the details of temperature dependence of the antisymmetrized signals in the Supplementary Fig. 12 and discuss the possible co-existence of different chiral types in $\mathrm{WS}_{2}$ NTs in the Supplementary Note 3) The oscillating terms, on the other hand, show the periodicity of $\phi_{0}$ (Fig. $4 \mathrm{c}-\mathrm{e}$ ) and enhancement around $T_{c}$ (Fig. $4 \mathrm{f}$ ) similar to the LP oscillations (Fig. 3b), indicating that both have the same origin. Interestingly, this term shows the stepwise behaviour at low temperature (Fig. 4e, from $\mu_{0} H=-1 \mathrm{~T}$ to $1 \mathrm{~T}$ ) distinct from the conventional linear relation of the external magnetic field according to equation (1). This analysis indicates that the nonreciprocal supercurrent also has the interference nature and is affected by the flux quanta passing through the NT.

\section{Discussion}

The observed second harmonic signals in AC resistance, together with LP oscillations in the first harmonic signals, are the direct manifestations of superconductivity in chiral NTs, however, the detailed mechanisms of the asymmetric electric transport and pairing symmetry (parity mixing) in the superconducting state needs to be further pursued. In light of this work, we expect various superconducting materials with broken inversion symmetry offer a similar transport, which provides a powerful 
approach for probing the exotic superconducting state in a variety of noncentrosymmetric systems.

\section{Methods}

Sample preparation. The $\mathrm{WS}_{2}$ NTs were synthesized following the literature ${ }^{22,23}$. The starting materials for the $\mathrm{WS}_{2} \mathrm{NT}$ synthesis route were spherical tungsten oxide nanoparticles, which were sulfurized by solid-gas reaction with hydrogen and hydrogen sulfide at elevated temperatures $\left(>800^{\circ} \mathrm{C}\right)$. During this one-pot reaction, tungsten suboxide whiskers grow and are subsequently sulfurized into $\mathrm{WS}_{2}$ NTs of $\sim 100 \mathrm{~nm}$ in diameter and up to 20 micron in length. These two main steps of the reaction-oxide whiskers growth and sulfurization-occur under the same $\mathrm{H}_{2} \mathrm{~S}_{\mathrm{H}} \mathrm{H}_{2}$ gas flow regime and are not separated in space following each other in a self-controlled mechanism.

Device fabrication. $\mathrm{WS}_{2} \mathrm{NTs}$ were dispersed in isopropyl alcohol solvent by ultrasonication for $20 \mathrm{~min}$. A droplet of the suspension was spin-coated on a $\mathrm{Si} / \mathrm{SiO}_{2}(3,000 \AA)$ substrate, and immediately covered by polymethyl methacrylate. The isolated $\mathrm{WS}_{2}$ NTs were subsequently chosen by an optical microscope. The device pattern was designed via standard electron beam lithography techniques and developed by mixed solution of methyl isobutyl ketone and isopropyl alcohol with the ratio of methyl isobutyl ketone:isopropyl alcohol $=1: 3$. After the deposition of $\mathrm{Cr} / \mathrm{Au}(5 \mathrm{~nm} / 90 \mathrm{~nm})$, pads and gate electrode were covered by photoresist and $\mathrm{Cr} / \mathrm{SiO}_{2}(5 \mathrm{~nm} / 20 \mathrm{~nm})$ was subsequently deposited to protect electrodes from the chemical reaction by ionic gating. Finally, we removed the redundant polymethyl methacrylate and gold by dipping the substrate into acetone for more than $1 \mathrm{~h}$. $\mathrm{KClO}_{4}$ /polyethylene glycol was selected as a gate medium ${ }^{19}$.

Transport measurements. All the transport properties have been measured in a Quantum Design Physical Property Measurement System with a horizontal rotator probe under He-purged and high-vacuum environments. High-vacuum mode was used when the temperature was higher than $200 \mathrm{~K}$, while the system was kept under He-purged condition below $200 \mathrm{~K}$. Gate voltage was applied by a Keithley 2400 sourcemeter at $300 \mathrm{~K}$ with a sweeping rate of $50 \mathrm{mV} \mathrm{s}^{-1}$ under high-vacuum condition. Both the first and the second harmonic signals of the AC resistance have been measured by a lock-in amplifier (Stanford Research Systems Model SR830 DSP) with a frequency of $13 \mathrm{~Hz}$. During the AC resistance measurements, the phase of the first (or second) harmonic signal was kept around 0 (or $\frac{\pi}{2}$ ), which is consistent with the theoretical expectation. All of the $R^{\omega}$ (or $R^{2 \omega}$ ) signals discussed in the main text were obtained as $\mathrm{x}$ (or $\mathrm{y}$ )-component of the lock-in measurement.

Data availability. All of the experimental data supporting this study are available from the corresponding author.

\section{References}

1. Rikken, G. L. J. A. \& Raupach, E. Observation of magneto-chiral dichroism. Nature 390, 493-494 (1997).

2. Berova, N., Nakanishi, K. \& Woody, R. W. Circular Dichroism: Principles and Applications 2nd edn (Wiley-VCH, 2000).

3. Nagaosa, N. \& Tokura, Y. Topological properties and dynamics of magnetic skyrmions. Nat. Nanotechnol. 8, 899-911 (2013).

4. Naaman, R. \& Waldeck, D. H. Spintronics and chirality: spin selectivity in electron transport through chiral molecules. Annu. Rev. Phys. Chem. 66, 263-281 (2015).

5. Rikken, G. L. J. A., Fölling, J. \& Wyder, P. Electrical magnetochiral anisotropy. Phys. Rev. Lett. 87, 236602 (2001).

6. Krstić, V., Roth, S., Burghard, M., Kern, K. \& Rikken, G. L. J. A. Magneto-chiral anisotropy in charge transport through sigle-walled carbon nanotubes. J. Chem. Phys. 117, 11315-11319 (2002).

7. Pop, F., Auban-Senzier, P., Canadell, E., Rikken, G. L. J. A. \& Avarvari, N. Electrical magnetochiral anisotropy in a bulk chiral molecular conductor. Nat. Commun. 5, 3757 (2014).

8. Iijima, S. Helical microtubules of graphitic carbon. Nature 354, 56-58 (1991).

9. Harris, P. J. F. Carbon Nanotube Science-Synthesis, Properties and Applications (Cambridge Univ. Press, 2009).

10. Rao, A. M. et al. Diameter-selective Raman scattering from vibrational modes in carbon nanotubes. Science 275, 187-191 (1997).

11. Bandow, S. et al. Effect of the growth temperature on the diameter distribution and chirality of single-wall carbon nanotubes. Phys. Rev. Lett. 80, 3779-3782 (1998).

12. Wildöer, J. W. G., Venema, L. C., Rinzler, A. G., Smalley, R. E. \& Dekker, C. Electronic structure of atomically resolved carbon nanotubes. Nature 391, 5962 (1998).

13. Kociak, M. et al. Superconductivity in ropes of single-walled carbon nanotubes. Phys. Rev. Lett. 86, 2416-2419 (2001).

14. Tang, Z. K. et al. Superconductivity in 4 angstrom single-walled carbon nanotubes. Science 292, 2462-2465 (2001).
15. Takesue, I. et al. Superconductivity in entirely end-bonded multiwalled carbon nanotubes. Phys. Rev. Lett. 96, 057001 (2006).

16. Shi, W. et al. Superconductivity in bundles of double-wall carbon nanotubes. Sci. Rep. 2, 625 (2012).

17. Wang, Q. H., Kalantar-Zadeh, K., Kis, A., Coleman, J. N. \& Strano, M. S. Electronics and optoelectronics of two-dimensional transition metal dichalcogenides. Nat. Nanotechnol. 7, 669-712 (2012).

18. Xu, X., Yao, W., Xiao, D. \& Heinz, T. F. Spin and pseudospins in layered transition metal dichalcogenides. Nat. Phys. 10, 343-350 (2014).

19. Shi, W. et al. Superconductivity series in transition metal dichalcogenides by ionic gating. Sci. Rep. 5, 12534 (2015).

20. Jo, S., Costanzo, D., Berger, H. \& Morpurgo, A. F. Electrostatically induced superconductivity at the surface of $\mathrm{WS}_{2}$. Nano Lett. 15, 1197-1202 (2015).

21. Tenne, R., Margulis, L., Genut, M. \& Hodes, G. Polyhedral and cylindrical structures of tungsten disulphide. Nature 360, 444-446 (1992).

22. Rothschild, A., Sloan, J. \& Tenne, R. Growth of $\mathrm{WS}_{2}$ nanotubes phases. J. Am. Chem. Soc. 122, 5169-5179 (2000).

23. Zak, A. et al. Scaling-up of the $\mathrm{WS}_{2}$ nanotubes synthesis. Fullerenes, Nanotubes and Carbon Nanostruct. 19, 18-26 (2010).

24. Levi, R., Bitton, O., Leitus, G., Tenne, R. \& Joselevich, E. Field-effect transistors based on $\mathrm{WS}_{2}$ nanotubes with high current-carrying capacity. Nano Lett. 13, 3736-3741 (2013).

25. Panchakarla, L. S. et al. Nanotubes from misfit layered compounds: a new family of materials with low dimensionality. J. Phys. Chem. Lett. 5, 3724-3736 (2014).

26. Rao, C. N. R. \& Nath, M. Inorganic nanotubes. Dalton Trans. 1, 1-24 (2003).

27. Bauer, E. \& Sigrist, M. Non-Centrosymmetric Superconductors: Introduction and Overview (Springer, 2012).

28. Gor'kov, L. P. \& Rashba, E. I. Superconducting 2D system with lifted spin degeneracy: mixed singlet-triplet state. Phys. Rev. Lett. 87, 037004 (2001).

29. Little, W. A. \& Parks, R. D. Observation of quantum periodicity in the transition temperature of a superconducting cylinder. Phys. Rev. Lett. 9, 9-12 (1962).

30. Li, L. J. et al. Controlling many-body states by the electric-field effect in a twodimensional material. Nature 529, 185-189 (2016).

\section{Acknowledgements}

We thank R. Popovitz-Biro for experimental help (HRTEM analysis) and R. Wakatsuki, M. Ezawa and N. Nagaosa for discussions. Y.I. acknowledges Grant-in-Aid for Specially Promoted Research (No. 25000003) from JSPS. T.I. acknowledges Grant-in-Aid for Research Activity Start-up (No.15H06133) from MEXT of Japan. R.T. acknowledges the support of Israel Science Foundation grant No. 265/12; the Kimmel Center for Nanoscale Science; the Perlman Family Foundation; the Irving and Azelle Waltcher Foundation in honour of Prof M. Levy. R.T. and A.Z. acknowledge the support of the FTA action No. 711543 of the Israel National Nano Initiative. A.Z. acknowledges the support of Pazi foundation.

\section{Author contributions}

F.Q., W.S., T.I. and Y.I. conceived and designed the experiments. A.Z. and R.T. performed the growth and characterization of the $\mathrm{WS}_{2}$ nanotubes. T.K., D.I. and D.H. carried out TEM experiments, SEM measurement and characterization. F.Q., W.S. and M.Y. fabricated electric double-layer transistor devices and measured transport properties. F.Q., T.I., M.Y. and Y.I. led the physical discussions. F.Q., T.I., A.Z., R.T. and Y.I. wrote the manuscript. All authors commented on the manuscript.

\section{Additional information}

Supplementary Information accompanies this paper at http://www.nature.com/ naturecommunications

Competing financial interests: The authors declare no competing financial interests.

Reprints and permission information is available online at http://npg.nature.com/ reprintsandpermissions/

How to cite this article: Qin, F. et al. Superconductivity in a chiral nanotube. Nat. Commun. 8, 14465 doi: 10.1038/ncomms14465 (2017).

Publisher's note: Springer Nature remains neutral with regard to jurisdictional claims in published maps and institutional affiliations.

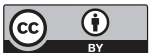

This work is licensed under a Creative Commons Attribution 4.0 International License. The images or other third party material in this article are included in the article's Creative Commons license, unless indicated otherwise in the credit line; if the material is not included under the Creative Commons license, users will need to obtain permission from the license holder to reproduce the material To view a copy of this license, visit http://creativecommons.org/licenses/by/4.0/

(C) The Author(s) 2017 